\section{Is There Really An Acute Alcohol-Related Axonal Polyneuropathy?}

To the Editor: We have read with interest the report by Sahin ${ }^{1}$ focusing on a possible alcohol-related polyneuropathy (ARP) with acute onset. It is well known that ARP usually affects sensory more than motor nerve fibers, has an insidious onset, and a chronic progressive course. Nevertheless, an acute axonal form has been proposed, which can be mistaken for Guillain-Barré syndrome (GBS). ${ }^{2} \mathrm{We}$ have recently seen one of those cases: a 36-year-old woman, with a 15-year history of excessive alcohol intake (90 g per day), was seen for a 3-week progressive leg weakness, shortly followed by distal paresthesias. Examination disclosed symmetrical distal and leg-predominant tetraparesis (upper extremities: proximal 5/5, distal 4/5; lower extremities: proximal $4 / 5$, distal $3 / 5)$, thermoalgic hypoesthesia in glove (fist) and stocking (knee) pattern; absent distal position sense, and deep tendon reflexes could not be elicited. Cerebrospinal fluid (CSF) at Day 1 and Day 20 were normal. Electromyography showed active innervation, with normal conduction velocities and no conduction blocks, suggesting an acute axonal polyneuropathy. The patient had normal lab values (including $\mathrm{CK}$, thyroid hormones, and liver enzymes). Anti-GM1 antibodies were negative. Sural nerve biopsy showed axonal degeneration, with no perivascular inflammatory changes or abnormal protein deposition. Under the clinical suspicion of GBS, she was treated with intravenous immunoglobulin. Remarkable recovery over the next 3 weeks was seen, and, 3 months later, with no further alcohol consumption, the neurologic examination and electromyography were completely normal. Although we can believe that this patient had an acute ARP, the very existence of this entity remains highly controversial for several reasons, which we found not to be adequately stressed in the Sahin letter: ${ }^{1}$ the better-known acute motor or motor and sensory neuropathy (AMAN/ AMSAN), the axonal GBS forms, can have a particularly benign course. ${ }^{2}$ Given that many cases, as in Walsh's ${ }^{3}$ description of seven patients with acute motor ARP (and others reviewed by Sahin), were reported before the description of AMAN/AMSAN, we do not know how they would be classified nowadays, ${ }^{3}$ alcohol intake is not systematically researched in GBS patients, all supporting features, such as normal CSF, absence of anti-GM1 antibodies, and demyelination on EMG or nerve biopsy, can occur in axonal GBS, the benign course should not be immediately related to abstinence, as it may result from immunoglobulin therapy or natural course. Because there is no report of recurrence of acute ARP, as would be expected in those who did not stop or resumed drinking, a direct, toxic, dose-related effect on nerve fibers is yet to be proved. This matter can only be disentangled if a well-designed clinical study shows that alcohol intake is associated with a benign acute axonal GBS, and if experimental studies prove definitely that axonal acute damage can be caused directly by ethanol. Until then, we think it is premature to assign to a putative alcohol-related direct effect what would otherwise be a more or less atypical axonal GBS.

MARgarida Rodrigues, M.D. SOFIA ROCHA, M.D.

Álvaro Machado, M.D.

António Guimarães, M.D. Neurology Department Hospital de Braga

Portugal

Neuropathology Unit

Hospital Geral de Santo

António

Porto, Portugal

Correspondence: Álvaro Alexandre Machado Salgado; e-mail: alvmac@gmail.com

\section{References}

1. Sahin S, Cinar N, Sezgin G, et al: Acute motor axonal neuropathy: a severe complication of alcohol abuse. J Neuropsychiatry Clin Neurosci 2010; 22:e16-e17

2. Wohrle JC, Spengos K, Steinke W, et al: Alcohol-related acute axonal polyneuropathy: a differential diagnosis of GuillainBarré syndrome. Arch Neurol 1998; 55:1329-1334

3. Walsh JC, McLeod JG: Alcoholic neuropathy: an electrophysiological and histological study. J Neurol Sci 1970; 10:457469 